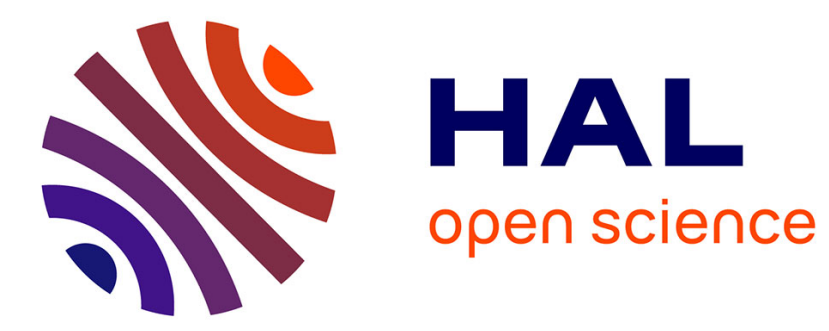

\title{
Large eddy simulation of decaying magnetohydrodynamic turbulence with dynamic subgrid-modeling
}

\author{
Olivier Agullo, W.-C. Müller, B. Knaepen, D. Carati
}

\section{To cite this version:}

Olivier Agullo, W.-C. Müller, B. Knaepen, D. Carati. Large eddy simulation of decaying magnetohydrodynamic turbulence with dynamic subgrid-modeling. Physics of Plasmas, 2001, 8 (7), pp.3502. 10.1063/1.1372337 . hal-01625879

\section{HAL Id: hal-01625879 \\ https://hal-amu.archives-ouvertes.fr/hal-01625879}

Submitted on 6 Feb 2018

HAL is a multi-disciplinary open access archive for the deposit and dissemination of scientific research documents, whether they are published or not. The documents may come from teaching and research institutions in France or abroad, or from public or private research centers.
L'archive ouverte pluridisciplinaire HAL, est destinée au dépôt et à la diffusion de documents scientifiques de niveau recherche, publiés ou non, émanant des établissements d'enseignement et de recherche français ou étrangers, des laboratoires publics ou privés. 


\title{
Large eddy simulation of decaying magnetohydrodynamic turbulence with dynamic subgrid-modeling
}

\author{
O. Agullo, ${ }^{\text {a) }}$ W.-C. Müller, ${ }^{\text {b) }}$ B. Knaepen, and D. Carati \\ Euratom-Belgian State Association, Physique statistique et des plasmas, CP 231, Campus Plaine, \\ Université Libre de Bruxelles, 1050 Bruxelles, Belgium
}

\begin{abstract}
The numerical large eddy simulation (LES) technique is tested on decaying magnetohydrodynamic (MHD) turbulence. The LES approach allows for a strong reduction in computational cost compared to direct numerical simulations by modeling the effects of the smallest turbulent scales instead of computing them directly. Two small-scale models of eddy-viscosity type are presented for this purpose in combination with a procedure for the self-consistent calculation of the model parameters in the course of the simulation. The method is successfully tested by comparing the obtained results to a high-resolution direct numerical simulation of decaying three-dimensional MHD turbulence.
\end{abstract}

Many plasmas in turbulent motion observed in astrophysical systems, as well as nuclear fusion devices, can be described within the framework of incompressible magnetohydrodynamics (MHD). Since direct experimental access to fully developed MHD turbulence is often difficult or even impossible, one resorts to the numerical simulation of idealized systems by solving the incompressible MHD equations

$$
\begin{aligned}
& \partial_{t} \mathbf{v}=-\nabla \cdot(\mathbf{v v}-\mathbf{b b})+\nu \Delta \mathbf{v}-\nabla p, \\
& \partial_{t} \mathbf{b}=-\nabla \cdot(\mathbf{v b}-\mathbf{b} \mathbf{v})+\eta \Delta \mathbf{b}, \\
& \nabla \cdot \mathbf{v}=\nabla \cdot \mathbf{b}=0,
\end{aligned}
$$

written here with the fluid velocity $\mathbf{v}$, the magnetic-field $\mathbf{b}$ expressed in Alfvén speed units (the constant mass density is rescaled to unity), the kinematic viscosity $\nu$, and magnetic diffusivity $\eta$. The dynamic pressure $p$ is obtained by imposing the incompressibility of $\mathbf{v}$.

Unfortunately, the numerical resolution necessary to render the discrete form of a turbulent MHD flow into a physically meaningful representation of the real system increases rapidly with the magnetic Reynolds number $R m=v_{0} l_{0} / \eta$, $v_{0}$ and $l_{0}$ being a characteristic flow velocity and length scale, respectively. This limits direct numerical simulations (DNS) of three-dimensional MHD turbulence on today's supercomputers $^{1-3}$ to values of $R m$ that are still several orders of magnitude lower than the ones found in nature, e.g., in the solar convection zone. A possible alternative to DNS

\footnotetext{
${ }^{a)}$ Present address: Equipe dynamique des systèmes complexes (case 321), Laboratoire PIIM, Université de Provence, Center universitaire de StJérôme, F13397-Marseille Cedex 20, France.

${ }^{b)}$ Electronic mail: wolf.mueller@ipp.mpg.de
}

is the large-eddy simulation (LES) approach, where the Reynolds number gap between simulation and reality is narrowed by restricting direct numerical computation to the large spatial scales of turbulence in combination with a model to incorporate the effects of the small-scale motions. This approximation is based on the assumption that the large-scale dynamics reflects the characteristic physics of a specific turbulent system, e.g., the influence of geometry and boundary conditions, while the small-scale turbulent fluctuations act as a statistically quasi-uniform background.

The LES technique is considered a valuable tool for simulating rather large Reynolds number Navier-Stokes turbulence for more than thirty years now, ${ }^{4}$ while work on adapting the method to MHD turbulence ${ }^{5-8}$ has not received as much attention. This might in part be caused by the fact that LES small-scale models usually contain at least one free parameter, which has to be tuned to obtain optimal performance of the simulation, turning a LES into a trial and error process. However, not long ago a dynamic procedure was developed $^{9}$ that allows for a self-consistent calibration of the small-scale model during the simulation, leading to convincing results when used in Navier-Stokes turbulence. In this Brief Communication we present the generalization and application of this procedure to isotropic three-dimensional MHD turbulence, applying two generalized eddy-viscosity models to describe the small-scale dynamics. Scalar-level tests are performed by comparing the observed temporal and spectral behavior of the kinetic and magnetic energies to the results of a high-resolution DNS of decaying MHD turbulence. $^{3}$

The formal basis of LES is the application of a spatial gridfilter of width $\bar{l}$ (Ref. 10) to (1)-(3). The system of fil- 
tered equations can then be solved using a coarser grid, since fluctuations on spatial scales smaller than $\bar{l}$ - the subgrid scales-have been removed. In the following, $\bar{l}$-filtered quantities will be denoted by an overbar. The filtered equations for $\overline{\mathbf{v}}$ and $\overline{\mathbf{b}}$ thus read

$$
\begin{aligned}
& \partial_{t} \overline{\mathbf{v}}=-\nabla \cdot\left(\overline{\mathbf{v}} \mathbf{\mathbf { v }}-\overline{\mathbf{b}} \overline{\mathbf{b}}+\tau^{\nu}\right)+\nu \Delta \overline{\mathbf{v}}-\nabla \bar{p}, \\
& \partial_{t} \overline{\mathbf{b}}=-\nabla \cdot\left(\overline{\mathbf{v}} \overline{\mathbf{b}}-\overline{\mathbf{b}} \overline{\mathbf{v}}+\tau^{b}\right)+\eta \Delta \overline{\mathbf{b}} \\
& \nabla \cdot \overline{\mathbf{v}}=\nabla \cdot \overline{\mathbf{b}}=0
\end{aligned}
$$

where two unknown terms, usually referred to as subgridscale or filtered-scale stress tensors, have been introduced by the filtering operation and need to be modeled: $\tau^{v}=(\overline{\mathbf{v v}}$ $-\overline{\mathbf{v}} \mathbf{v})-(\overline{\mathbf{b} b}-\overline{\mathbf{b}} \overline{\mathbf{b}})$ and $\tau^{b}=(\overline{\mathbf{v b}}-\overline{\mathbf{v}} \overline{\mathbf{b}})-(\overline{\mathbf{b} \mathbf{v}}-\overline{\mathbf{b}} \overline{\mathbf{v}})$. These tensors account for effects of the subgrid-scale motions on the flow at the resolved scales larger than $\bar{l}$. The filter chosen here is a sharp cubic Fourier cutoff, which sets all spatial Fourier modes with at least one wave-vector component larger than $k_{c}=\pi / \bar{l}$ to zero.

In order to assess the LES technique and the dynamic procedure in MHD we have performed simulations of decaying isotropic turbulence using the same pseudospectral method as the reference DNS, the same integration domain (a cube of linear extension $2 \pi$ with periodic boundary conditions) and the same set of parameters $\left(\nu=\eta=3 \cdot 10^{-4}\right)$ at a significantly reduced numerical resolution. The initial condition has been generated from the reference DNS data at $t$ $=1$ when kinetic and magnetic energy dissipation, starting from smooth initial fields, have reached their maxima and the dissipative small-scale structures of turbulence are fully developed. The data set has been cutoff-filtered from $512^{3}$ to $64^{3}$ Fourier modes, removing about $99.8 \%$ of the originally available information. The influence of the filtered scales on the remaining large scales of motion, which still contain $90 \%$ of the total energy, has to be mimicked by the models for the filtered-scale stress tensors $\tau^{\nu}$ and $\tau^{b}$.

The model expressions used here are based on the eddyviscosity assumption widely applied in LES of NavierStokes turbulence. ${ }^{11}$ This class of models aims at reproducing the subgrid-scale kinetic-energy dissipation by assuming a linear relation between the deformation tensors at resolved and dissipative scales

$$
\boldsymbol{\tau}^{\Downarrow}=-2 \nu_{t} \overline{\mathbf{S}}, \quad \overline{\mathbf{S}}=\left(\nabla \overline{\mathbf{v}}+[\nabla \overline{\mathbf{v}}]^{T}\right) / 2,
$$

where the superscript " $T$ " denotes the transposed matrix and $\nu_{t}$ is the eddy-viscosity. When restricting the dependence of $\nu_{t}$ to a characteristic mixing-length $\bar{l}$ on the spatial scales under consideration and the subgrid kinetic-energy dissipation $\varepsilon^{K}$, dimensional analysis suggests $\nu_{t} \sim \bar{l}^{4 / 3}\left(\varepsilon^{K}\right)^{1 / 3}$. For the magnetic energy one can proceed analogously, taking into account that the property of the magnetic field, which gives rise to resistive dissipation, is its curl rather than its deformation. This yields the model prototype

$$
\tau^{b}=-2 \eta_{t} \overline{\mathbf{J}}, \quad \overline{\mathbf{J}}=\left(\nabla \overline{\mathbf{b}}-[\nabla \overline{\mathbf{b}}]^{T}\right) / 2,
$$

with the eddy-resistivity $\eta_{t} \sim \bar{l}^{4 / 3}\left(\varepsilon^{M}\right)^{1 / 3}, \varepsilon^{M}$ denoting the subgrid magnetic energy dissipation.

Depending on the way of estimating the subgrid dissipation, different eddy-viscosity models can be constructed. Here we consider two variants. If $\bar{l}$ lies somewhere in the inertial scale range of fully developed turbulence and, as observed in the reference DNS, the nonlinear energy transfer between kinetic and magnetic energy is much smaller than the respective energy dissipation, $\varepsilon^{K}$ and $\varepsilon^{M}$ can be assumed as spatially constant. This yields the generalized Kolmogorov scaling subgrid model

$$
M_{1}: \quad \nu_{t}=C_{1}(t) \bar{l}^{-4 / 3}, \quad \eta_{t}=D_{1}(t) \bar{l}^{-4 / 3},
$$

where $C_{1}$ and $D_{1}$ are unknown parameter functions of time.

The widely applied Smagorinsky model ${ }^{12}$ approximates $\varepsilon^{K}$ as the product of the resolved local dissipation rate and the associated length scale $\bar{l}$, leading to $\varepsilon^{K} \sim \bar{l}^{2}(2 \mathbf{S}: \mathbf{S})^{3 / 2}$. Together with the corresponding extension to $\mathrm{MHD}^{8} \varepsilon^{M}$ $\sim \bar{l}^{2}|\overline{\mathbf{j}}|^{3}, \overline{\mathbf{j}}=\nabla \times \overline{\mathbf{b}}$ being the resolved electric current density, this gives

$$
M_{2}: \quad \nu_{t}=C_{2}(t) \bar{l}^{2}(2 \mathbf{S}: \mathbf{S})^{1 / 2}, \quad \eta_{t}=D_{2}(t) \bar{l}^{2}|\overline{\mathbf{j}}| .
$$

Both models contain parameters $C_{i}$ and $D_{i}(i=1,2)$, for which no direct relationship with the resolved large-scale quantities can be obtained without further assumptions. Therefore, one would be forced to prescribe these parameters in order to optimize the simulation results with respect to known reference characteristics. Apart from the arbitrariness introduced to the subgrid-modeling by such an approach, the lack of sufficiently large experimental MHD turbulence databases, like they exist for the Navier-Stokes case, prevents any a priori justification of freely prescribed subgrid-model parameters. Fortunately, this problem can be circumvented by exploiting the self-similarity of fully developed turbulence. A corresponding technique, that has proven to be successful in Navier-Stokes LES, is the dynamic procedure. $^{13-16}$

The basic idea of this method is the application of a second filter - the test filter — of width $\hat{l}>\bar{l}$ to the LES equations (4)-(6). The test filtering operation, here a cubic cutoff with $\hat{l}=2 \bar{l}$, will be indicated by the hat symbol, $\hat{\bullet}$. Introducing the filtered-scale stress tensors at test filter level as $\mathbf{T}^{v}$ $=\left(\widehat{\widehat{\mathbf{v v}}}-\hat{\overline{\mathbf{v}}} \hat{\overline{\mathbf{v}}}-(\widehat{\mathbf{b b}}-\hat{\overline{\mathbf{b}}} \hat{\overline{\mathbf{b}}})\right.$ and $\mathbf{T}^{b}=(\widehat{\mathbf{v b}}-\hat{\overline{\mathbf{v}}} \hat{\overline{\mathbf{b}}})-(\widehat{\widehat{\mathbf{b v}}}-\hat{\overline{\mathbf{b}}} \hat{\overline{\mathbf{v}}})$ leads to the Germano identities ${ }^{14}$ for MHD

$$
\mathbf{T}^{v}=\hat{\boldsymbol{\tau}}^{v}+\mathbf{L}^{v}, \quad \mathbf{T}^{b}=\hat{\boldsymbol{\tau}}^{b}+\mathbf{L}^{b},
$$

where the Leonard tensors $\mathbf{L}^{v}=(\widehat{\mathbf{v v}}-\hat{\mathbf{v}} \hat{\mathbf{v}})-(\widehat{\mathbf{b b}}-\hat{\overline{\mathbf{b}}} \hat{\overline{\mathbf{b}}})$ and $\mathbf{L}^{b}=(\widehat{\mathbf{v b}}-\hat{\hat{\mathbf{v}}} \hat{\overline{\mathbf{b}}})-(\widehat{\mathbf{b v}}-\hat{\overline{\mathbf{b}}} \hat{\overline{\mathbf{v}}})$ are known in terms of the resolved fields and, as such, do not require any modeling. In contrast, the filtered-scale stress tensors at grid scales $\left(\tau^{\nu}, \tau^{b}\right)$ and test scales $\left(\mathbf{T}^{v}, \mathbf{T}^{b}\right)$ have to be modeled. Since the turbulent fields are assumed to be self-similar on scales within the interval $[\bar{l}, \hat{l}]$, the applied models and their parameters are independent of the test filter width. Hence, for the stress tensors $\mathbf{T}^{v}$ and $\mathbf{T}^{b}$ at test filter level the same model and coefficients should be used as for $\tau^{v}$ and $\tau^{b}$, respectively. 


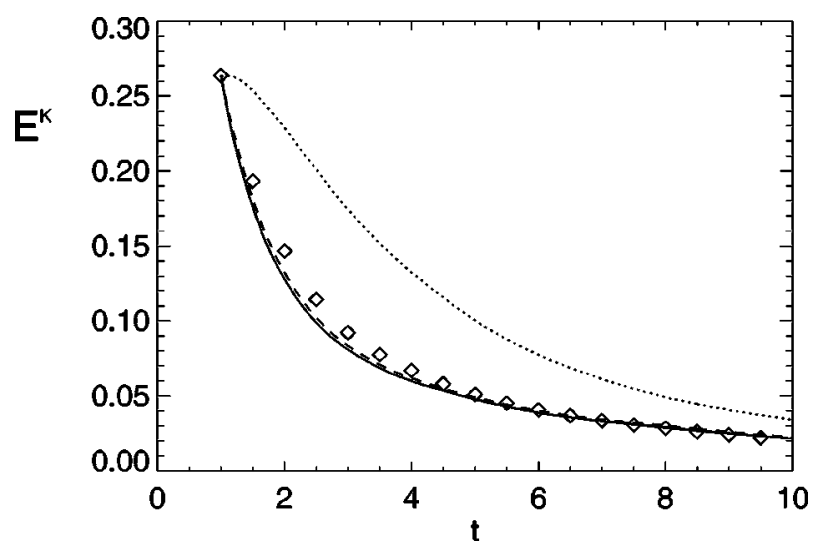

FIG. 1. Decay of the kinetic-energy $E^{K}$. Diamonds correspond to the reference DNS filtered to $64^{3}$, the dotted line shows the result of the $M_{0}$-LES without model. The solid and dashed lines represent the predictions of the LES with, respectively, model $M_{1}$ and model $M_{2}$.

Due to the modeling of the filtered-scale stress tensors the identities (7) are not exactly fulfilled. The errors can be quantified as

$Q^{v}=\int_{V} d^{3} r\left(\mathbf{T}^{v}-\hat{\boldsymbol{\tau}}^{v}-\mathbf{L}^{v}\right)^{2}, \quad Q^{b}=\int_{V} d^{3} r\left(\mathbf{T}^{b}-\hat{\boldsymbol{\tau}}^{b}-\mathbf{L}^{b}\right)^{2}$,

where the model parameters are assumed to be constant in space, which is justified when dealing with spatially homogeneous turbulence. Minimizing the deviations $Q^{v}$ and $Q^{b}$ by variation with respect to the model coefficients ${ }^{13}$ yields an optimization ansatz for the filtered scale stress tensors. The implementation of the dynamic procedure is somewhat more difficult when there is no flow direction of homogeneity. However, several solutions have been considered in that case, like the local dynamic procedure ${ }^{17}$ or the Lagrangian dynamic procedure. ${ }^{18}$ The choice of the ratio $\alpha=\hat{l} / \bar{l}=2$ as mentioned above has been guided by a series of test runs with model $M_{1}$, confirming the self-similarity of the turbulent fields for $\alpha \in[1.5,2.5]$.

A basic LES requirement is the reproduction of the temporal and spectral behavior of the filtered kinetic $\left(E^{K}\right)$ and magnetic $\left(E^{M}\right)$ energy. The time evolution of these quantities is shown in Figs. 1 and 2, where $t$ is given, like for the reference DNS, in units of the large eddy turnover time. Diamonds represent the filtered DNS results, the solid and the

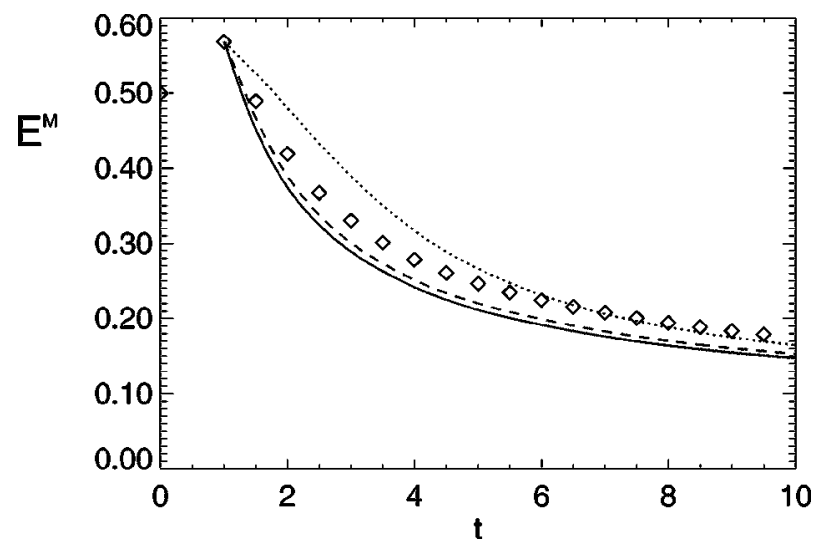

FIG. 2. Decay of the magnetic energy $E^{M}$. For symbol explanation see Fig. 1.

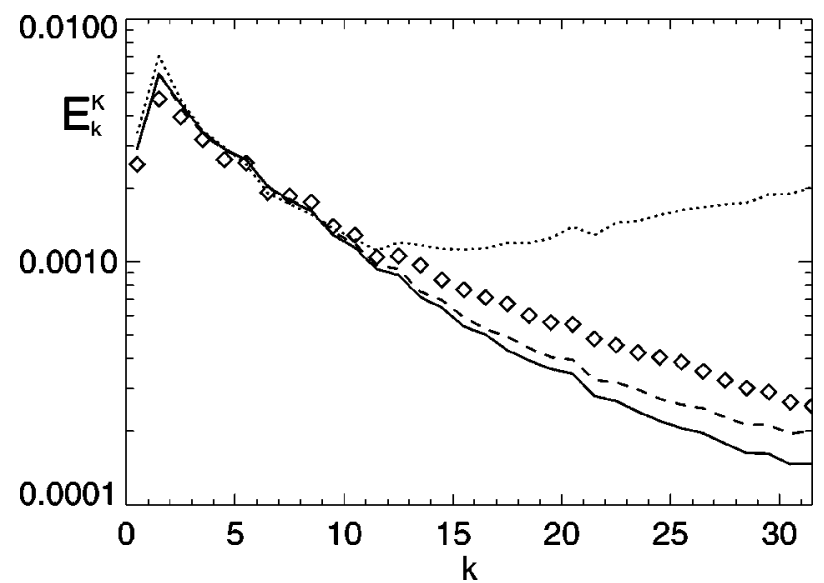

FIG. 3. Kinetic-energy spectrum $E_{k}^{K}$ at time $t=6$. Symbols are the same as in Fig. 1.

dashed lines give the energy development for LES with the models $M_{1}$ and $M_{2}$, respectively. To evaluate the overall influence of the dynamic subgrid modeling the dotted lines show the energy curves for a LES with $M_{0}: \tau^{v}=\tau^{b}=0$.

As expected, the $M_{0}$-LES results strongly deviate from the filtered DNS data, since energy dissipation is mainly due to the high wave number modes, which have been cutoff by the gridfiltering operation. The lack of a well-developed dissipation range hampers energy decay and leads to an accumulation of kinetic and magnetic energy in the high wave number region of the LES system (see below).

A clear improvement is observed when the subgrid models $M_{1}$ and $M_{2}$ are applied. The evolution of $E^{K}$ is well reproduced in both cases, showing that the main influence of the small-scale velocity field fluctuations on the kinetic energy is dissipative. The temporal development of the magnetic energy in the LES with $M_{1}$ and $M_{2}$ also satisfactorily fits the reference data, though one observes an offset between the LES results and the DNS. Closer inspection reveals that this phenomenon is caused by an initial overshoot of the model resistivities estimated by the dynamic procedure. This effect is known from Navier-Stokes LES using dynamic models and can be reduced by allowing for a short model relaxation period at the beginning of the simulation. Due to the spatial homogeneity of the turbulent system, there is only a small systematic difference between the results obtained with the Kolmogorov scaling model $M_{1}$, which mainly depends on the spatially averaged extrapolation process inherent to the dynamic procedure, and the Smagorinsky version $M_{2}$, that additionally takes into account the local field gradients.

The application of the virtually parameter free dynamic subgrid models reproduces rather sensitive quantities like the angle-averaged energy spectra in good agreement with the DNS data. The kinetic-energy spectra $E_{k}^{K}$ are shown in Fig. 3 at time $t=6$, when $E^{K}$ has decreased by a factor of about 6.5 . Both models, $M_{1}$ and $M_{2}$, lead to spectra that follow the filtered DNS data up to the high wave number range of the LES system. Nevertheless, the simple applied eddy-viscosity models provide too much dissipation in the high wave number range, leading to a slightly stronger fall-off of the LES spectra when compared to the reference data. One observes 


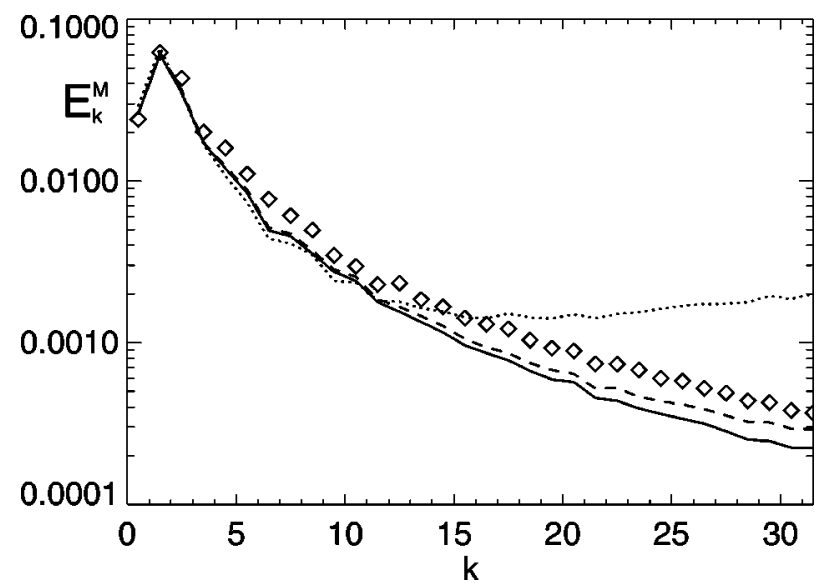

FIG. 4. Magnetic-energy spectrum $E_{k}^{M}$ at time $t=6$. Symbols are the same as in Fig. 1.

that the over-dissipation is reduced by the Smagorinsky term in model $M_{2}$, yielding a better spectral performance.

The agreement of the LES results with the filtered DNS spectrum is evidently due to the subgrid models $M_{1}$ and $M_{2}$, since the lack of such a model in the $M_{0}$-LES causes a large accumulation of kinetic energy over nearly two-thirds of the LES wave number space, which is the consequence of the missing filtered-scale energy dissipation in combination with the direct spectral energy cascade.

The same trends are observed in Fig. 4 for the angleaveraged magnetic energy spectrum $E_{k}^{M}$. However, the LES show a spectral magnetic-energy distribution that is too low across a wide range of scales when compared to the DNS spectrum. Since the $M_{0}$-LES without model exhibits the same behavior, the observation cannot be explained by the subgrid models being too dissipative. This implies that the model for the filtered-scale stress tensor $\tau^{b}$ in Eq. (5) has to be more complex than the simple dissipative subgrid models $M_{1}$ and $M_{2}$, e.g., allowing for a spectrally local inflow of magnetic energy. Nevertheless, the improvement in reproducing the magnetic energy reference spectrum using the presented subgrid-scale models compared to a $M_{0}$-LES is still tremendous.
The results presented in this Brief Communication show that LES with dynamic subgrid modeling are able to reproduce the temporal and spectral energy evolution in isotropic MHD turbulence. Since decaying turbulence was considered, the LES subgrid models had to adapt to a nonstationary environment, where a continuous self-calibration of the model parameters as realized in the dynamic procedure is of particular importance. The achieved LES performance, especially when the highly reduced numerical resolution and the simplicity of the purely dissipative filtered-scale models are taken into account, encourages further work on dynamic subgrid modeling of MHD turbulence.

\section{ACKNOWLEDGMENTS}

D.C. and B.K. are, respectively, "Chercheur Qualifié", and "Chargé de recherche" of the "Fonds National de la Recherche Scientifique’, (Belgium).

${ }^{1}$ M.-M. Mac Low, R. S. Klessen, A. Burkert, and M. D. Smith, Phys. Rev. Lett. 80, 2754 (1998).

${ }^{2}$ J. M. Stone, E. C. Ostriker, and C. F. Gammie, Astrophys. J. Lett. 508, L99 (1998).

${ }^{3}$ D. Biskamp and W.-C. Müller, Phys. Plasmas 7, 4889 (2000).

${ }^{4}$ R. Rogallo and P. Moin, Annu. Rev. Fluid Mech. 16, 99 (1984).

${ }^{5}$ A. Yoshizawa, Phys. Fluids 30, 1089 (1987).

${ }^{6}$ T. Passot, H. Politano, A. Pouquet, and P. L. Sulem, Theor. Comput. Fluid Dyn. 1, 47 (1990).

${ }^{7}$ Y. Zhou and G. Vahala, J. Plasma Phys. 45, 239 (1991).

${ }^{8}$ M. Theobald, P. Fox, and S. Sofia, Phys. Plasmas 1, 3016 (1994).

${ }^{9}$ M. Germano, U. Piomelli, P. Moin, and W. H. Cabot, Phys. Fluids A 3, 1760 (1991).

${ }^{10}$ A. Leonard, Adv. Geophys. 18A, 237 (1974).

${ }^{11}$ D. Leslie and G. Quarini, J. Fluid Mech. 91, 65 (1979).

${ }^{12}$ J. Smagorinsky, Mon. Weather Rev. 91, 99 (1963).

${ }^{13}$ D. Lilly, Phys. Fluids A 4, 633 (1992).

${ }^{14}$ M. Germano, J. Fluid Mech. 238, 325 (1992).

${ }^{15} \mathrm{P}$. Moin and J. Jimenéz, in AIAA 24th Fluid Dynamics Conference, Orlando, Florida, AIAA 93-3099 (American Institute of Aeronautics and Astronautics, Washington, DC, 1993).

${ }^{16}$ D. Carati, S. Ghosal, and P. Moin, Phys. Fluids 7, 606 (1995).

${ }^{17}$ S. Ghosal, T. Lund, P. Moin, and K. Akselvoll, J. Fluid Mech. 286, 229 (1995).

${ }^{18}$ C. Meneveau, T. Lund, and W. Cabot, J. Fluid Mech. 319, 353 (1996). 